\title{
A review of Entoloma sensu lato (Basidiomycota, Entolomataceae) from Yunnan Province, China
}

\section{Ediriweera $\mathrm{AN}^{2,3,4,5}$, Karunarathna $\mathrm{SC}^{1,2,3,4}$, Xu J $\mathrm{J}^{1,2^{*}}$, Hyde $\mathrm{KD}^{1,2,3}$, Raspé $\mathrm{O}^{2}$ and De Silva NI $^{2}$}

${ }^{1}$ CAS Key Laboratory for Plant Diversity and Biogeography of East Asia, Kunming Institute of Botany, Chinese Academy of Sciences, Kunming 650201, Yunnan, China

${ }^{2}$ Centre for Mountain Ecosystem Studies, Kunming Institute of Botany, Chinese Academy of Sciences, Kunming 650201, China

${ }^{3}$ Center of Excellence in Fungal Research, and School of Science, Mae Fah Luang University, Chiang Rai 57100, Thailand

${ }^{4}$ World Agroforestry Centre, East and Central Asia, 132 Lanhei Road, Kunming 650201, China

${ }^{5}$ Department of Biosystems Technology, Faculty of Technology, University of Ruhuna

Ediriweera AN, Karunarthna SC, Xu J, Hyde KD, Raspé O, De silva NI 2020 - A review on the genus Entoloma (Basidiomycota, Entolomataceae) in Yunnan Province, China. Studies in Fungi 5(1), 526-535, Doi 10.5943/sif/5/1/33

\begin{abstract}
This review succinctly discusses data on morphology, systematics, ecology and biochemical properties of all the Entoloma species from Yunnan Province, China. We also propose directions for future research on Entoloma. Altogether, 8 species of Entoloma have been reported in the present study. Entoloma sinuatum and E.mengsongense showed the most contrasting variation in colour and size of the basidiomes while E. mengsongense and E. yunnanense were found to display wide variations in spore morphology. Most species are saprotrophic except E. caeruleoflavum and $E$. sinuatum which form mycorrhizal associations. Knowledge on biochemical and other chemical attributes of the genus are minimal although such details would help for better utilization of the genus.
\end{abstract}

Keywords - Checklist - Ecology - macrofungi - morphology - phylogeny

\section{Introduction}

Entoloma (Fr.) P. Kumm. (1871), belonging in Entolomataceae, is known from a wide range of geographic domains including the tropics and Arctic regions (Hesler 1967, Largent 1994, Manimohan et al.1995, 2006, Noordeloos 2004, Gates \& Noordeloos 2007, Noordeloos \& Hausknecht 2007, Co-David et al. 2009, Karstedt \& Capelari 2013). Almost 1500 Entoloma species are known worldwide (Gates \& Noordeloos 2009, Noordeloos \& Morozova 2010, He et al. 2012, $\mathrm{He}$ et al. 2019b) the majority of which are saprotrophic, though a few species like $E$. caeruleoflavum (Xiao HL \& Li TH) E. sepium (Noulet \& Dassier) Richon \& Roze, E. aprile (Britzelm.) Sacc., E. saundersii (Fr.) Sacc., E.sinuatum (Pers.) P. Kumm. (1871) \& E. clypeatum (L.) P. Kumm. have been shown to be mycorrhizal (Co-David et al. 2009). Entoloma species can be seen in a wide range of habitats such as rainforests, grasslands, temperate to alpine forests and peatbogs (Zhang \& Li 2001b, Co-David et al. 2009, Gates et al. 2009, Kasuya et al. 2010, Gargano et al. 2011, He et al 2019b). 


\section{Entoloma in China}

During the past two decades there has been an increased interest in the diversity of Entoloma in China, with several new species being described (Zhang \& Li 2001a, Li et al. 2009, Li \& Li 2009). The first Entoloma species described from China was E. quadratum (Berk. \& M.A. Curtis) E. Horak. Prior to this, about twenty cuboid spored macrofungi species had been reported (Xiao et al. 2015) and later number of Entoloma species were reported from a wide range of habitats and ecosystems ( $\mathrm{Li}$ et al. 2009, Li \& Li 2009, He et al. 2010).

Increased research carried out in China on the family Entolomataceae, several new Entoloma species have been subsequently described (He et al. 2012, Xiao et al. 2015, Ediriweera et al. 2017). E. caeruleoflavum Xiao LH \& Li TH, E. hainanense Li TH \& Xiao LH, and E. subtenuicystidiatum Xiao LH (He et al. 2012) from southern China and E. mengsongense Ediriweera et al. from Mengsong, Yunnan (Ediriweera et al. 2017) have described recently from Yunnan.

DNA-based identification is on the upsurge in macrofungi with recent development and availability of facilities while the morphology based identification is still followed as the standard methods for identification. Hence both molecular level identification and morphological identification is significant for the precision of macrofungi identification. In Entoloma various macromorphological features viz., size, shape, colour, texture, odour, taste etc of basidiomata are crucial for species identification. Habit, habitat and ecological factors are also important in delimiting species in Entoloma.

Yunnan Province is a low altitude plateau with multiplex topography and variable climate, which are ideal conditions for a high diversity of macrofungi, including Entoloma. The weather of most areas of Yunnan province is reasonably mild in winter and slightly cool in summer. Yunnan province is known as warm region in China where average daily temperature is $24^{\circ} \mathrm{C}$ and often with several months of warm and humid weather (World Data Lab 2020).

This review intends to update the information on Entoloma species described from or reported from Yunnan Province, China. The data will eventually help and pave way for better understanding of the species diversity of Entoloma in the region, which in turn will help to formulate conservation measures.

\section{Checklist of Entoloma species in Yunnan province, China}

The spore quotient is denoted by "Q" in each micro-morphological description of species.

Entoloma caeruleoflavum Xiao L. He \& T.H. Li, in He et al. Mycol. Progress 11: 4 (2012)

Following description is based on literature based on research publication of He et al. 2012

Macro-morphology - Basidiomata moderate size. Pileus 30-60 $\mathrm{mm}$ in diameter, planoconvex, expanding with maturity, minutely scurfy, blackish blue, outwards paler and mixed with green, dry, not hygrophanous, sulcate from margin to half-center and undulate at margin. Lamellae sinuate, ventricose, up to $8 \mathrm{~mm}$ broad, thin, crowded, bright yellow that turns pink to reddish brown when bruised, wavy and edge, three tiers of lamellulae. Stipe central, cylindrical, 50-100 × 4-10 $\mathrm{mm}$, broadening towards base, dark blue, longitudinally fibrillose, hollow, fragile, dry, white tomentum at basal area. Thin Context, whitish. Odor and taste not distinctive.

Micro-morphology - Basidiospores 6.5-7.5 × 6.3-7.3 $\mu \mathrm{m}, \mathrm{Q}=1.0-1.1$, isodiametric, 5-7 angled in side-view, thin walled. Basidia 30-48 $\times 9-12 \mu \mathrm{m}$, clavate, tapered at base, 4-spored or 2spored, clamped. Lamellar trama subparallel, composed of inflated and cylindrical elements, 40-90 $\times 8-22 \mu \mathrm{m}$. Fertile Lamellar edge. Cheilocystidia, pleurocystidia absent. Pileipellis a cut is showing a transition to a trichoderm with interwoven hyphae where terminal cells are differentiated, subclavate, bullet-shaped, inflated, 27-63×11-16 $\mu \mathrm{m}$, conspicuous brown intracellular pigment. Pileitrama regular, formed with short slightly inflated elements, $23 \mu \mathrm{m}$ broad, pale pink intracellular pigment or sometimes nearly hyaline. Clamp connections present. Oleiferous hyphae visible in lamellar trama and pileal trama.

Ecology and Distribution - Mycorrhizal, scattered, on soil in mixed forest with Fagaceae, Theaceae and Pinus yunnanensis. Recorded from Yunnan, China (He et al. 2012). 
Entoloma conchatum Xiao L. He \& E. Horak, in He et al. MycoKeys 61:1-26 (2019a)

Following description is based on literature gathered from publication of He et al. 2019a which was the protologue for this species

Macro-morphology - Basidiomata moderate size. Pileus 7-15 mm in diameter, conchate and broadly convex, initially white colour, turns orange-white or yellowish-white and ultimately turns in to pale pinkish with maturity, entirely matted-tomentose, opaque, dry, fibrillose, not hygrophanous, margin is not transparent-striate. Lamellae adnexed, $2 \mathrm{~mm}$ wide, subdistant, subventricose, consists of 2-4 tiers of lamellulae, white colour at first, turning pinkish with maturity, margin concolorous. Stipe short, 1-3 × 0.5-1 mm, strongly, lateral, strongly reduced, covered with minute, white fibrils, base with white mycelium. Rhizoids absent. Context white, thin, unchanging. Odour and tastenot distinctive.

Micro-morphology - Basidiospores: 8-10 $\times$ 6.5-8 $\mu \mathrm{m}, \mathrm{Q}=$ 1.2-1.4, 5-6-angled, heterodiametric in profile view. Basidia 28-34 ×9-12 $\mu \mathrm{m}, 4$-spored or 2-spored, subclavate, fertile lamellar edge. Cheilocystidia, pleurocystidia, caulocystidia absent. Pileipellis a cutis formed of cylindrical hyphae, terminal cells $35-50 \times 4-7 \mu \mathrm{m}$, cylindrical, poorly gelatinised wall thin, minutely encrusted light yellow pigments. Oleiferous hyphae present in pileipellis. Clampconnections abundant in all tissues.

Ecology and distribution - saprotrophic, grows on moss of stem basal area of live conifers and fallen branches of conifers, Recorded from Yunnan, China (He et al. 2019a).

Entoloma gregarium Xiao L. He \& E. Horak, in He et al. MycoKeys 61: 1-26 (2019a)

Following description is based on literature gathered from publication of He et al. 2019a which was the protologue for this species.

Macro-morphology - Basidiomata small. Pileus 5-10 $\mathrm{mm}$ in diameter, broadly convex shape, white colour, no colour change with maturity, entirely matted-tomentose, fibrillose, dry, opaque, not hygrophanous, margin not striate. Lamellae adnexed, sub-distant to distant, sub-ventricose, 2 $\mathrm{mm}$ wide, two tiers of lamellulae present, white colour when young later become pale pink, in the presence of moist with small red droplets at edges. Stipe $1-3 \times 0.5-1 \mathrm{~mm}$, lateral, strongly reduced, translucent, covered with white fibrils, white basal mycelium. White context, thin. No distinctive Odour and taste.

Micro-morphology - Basidiospores 7-9 $\times$ 5.5-7 $\mu \mathrm{m}, \mathrm{Q}=1.16-1.47,5-6$ angled, heterodiametric in profile view. Basidia 4-spored, 30-34 × 7-10 $\mu \mathrm{m}$, subclavate, clampless. Pleurocystidia, Cheilocystidia and caulocystidia absent. Pileipellis a cutis of cylindrical hyphae, terminal cells $35-60 \times 5-10 \mu \mathrm{m}$, subclavate or cylindrical, repent or slightly uplifted, nongelatinised thin wall, smooth, with inconspicuous plasmatic pigment, subpellis formed with shortcelled cylindrical hyphae, Oleiferous hyphae of 6-14 $\mu \mathrm{m}$ present in pileipellis. Clamp-connections present in all tissues.

Ecology and Distribution - Saprotrophic, grow on moss on stem base of Castanopsis in fagalean forest. Recorded from Yunnan, China.

Entoloma mengsongense Ediriweera, Karun., J.C. Xu, K.D. Hyde \& P.E. Mortimer, Turkish Journal of Botany 41 (5): 509 (2017)

Following description is based on literature gathered from publication of Ediriweera et al. 2017 which was the protologue for this species.

Macro-morphology - Basidiomata large. Pileus 3.5-5 cm in diameter, conical when young, plano-concave when mature, umbo in center, dry surface, fibrillose, more fibrillose near center, yellowish brown surface due to the fibrillose pileipellis. Lamellae adnexed, moderately crowded with lamellulae of 4 lengths. Stipe 65-90 × 6-7 mm, cylindrical, brittle, solid, surface fibrillose, concolorous when young and turns sky blue at maturity. Context white, thin, greenish blue or yellowish green when bruised. Mycelium cottony appearance at the base. Odor peculiar and distinct (Ediriweera et al. 2017). 
Micro-morphology - Spores 4-8 $\times 4-6 \mu \mathrm{m}$, cuboid-quadrate, regular or irregular. Basidia 40-60 × 9-14 $\mu \mathrm{m}$, clavate/obclavate, 1-4 spored, sterigmata $5 \mu \mathrm{m}$ long. Cheilocystidia 35-40 $\times 9$ $12 \mu \mathrm{m}$, thin-walled, clavate and hyaline. Hymenophoral trama is subregular, hyphae 3-15 $\mu \mathrm{m}$ diameter, thin-walled, hyaline. Subhymenium poorly developed. Pileal trama parallel and interwoven. Pileipellis a cutis, hyphae $2-10 \mu \mathrm{m}$ diameter, thin-walled, pale yellowish. Stipitipellis a disrupted cutis, hyphae 1-8 $\mu \mathrm{m}$ wide, hyaline, thin-walled. They form either ascending or erect bundles, clavate or cylindrical hyphae, $7-8.5 \mu \mathrm{m}$ diam. Clamp connections present in all tissues (Ediriweera et al. 2017).

Ecology and Distribution - Saprotrophic. In small groups on soil, known only from the type locality in Yunnan, China (Ediriweera et al. 2017).

Entoloma pleurotoides Xiao L. He \& E. Horak in He et al. MycoKeys 61: 1-26 (2019a)

Following description is based on literature gathered from publication of He et al. 2019a which was the protologue for this species.

Macro-morphology - Basidiomata small. Pileus 5-15 mm, conchate, broadly convex when young and become applanate when mature, entirely matted- tomentose, membranous, fibrillose, white at first, turns orange, yellowish-white and pale pinkish at maturity, hygrophanous, margin not transparent-striate. Lamellae adnexed, lamellulae with 1-2 tiers, narrow, distant, $1.5 \mathrm{~mm}$ wide, subventricose, white, pinkish with maturity, entire edges are concolorous. Stipe $1-2.5 \times 0.5-1 \mathrm{~mm}$, lateral, reduced, light grey brownish, slightly covered with pale greyish fibrils, white mycelium present at base. Thin Context. Odour absent. Indistinctive taste.

Micro-morphology - Basidiospores 8-10 $\times$ 7.5-9.5 $\mu \mathrm{m}, \mathrm{Q}=1.0-1.25,5-6$ angled, isodiametric to subisodiametric. Basidia 4-spored, 32-40 × 12-14 $\mu \mathrm{m}$, clavate, clampless. Fertile Lamellar edge. Cheilocystidia, pleurocystidia, caulocystidia absent. Pileipellis a cutis formed with cylindrical hyphae, terminal cells 30-40 × 3-8 $\mu \mathrm{m}$, either subclavate or cylindrical, slightly uplifted, thin, non-gelatinised wall, smooth, plasmatic pigment present, subpellis formed with short-celled cylindrical hyphae, 5-10 $\mu \mathrm{m}$ of diameter. Oleiferous hyphae present. Clampconnections present.

Ecology and Distribution - Saprotrophic, grow on moss at base of living Castanopsis sp. and sometimes on debris of Castanopsis sp. Recorded from Yunnan, China (He et al.2019a).

Entoloma reductum Xiao L. He \& E. Horak in He et al. MycoKeys 61: 1-26 (2019a)

Following description is based on literature gathered from publication of He et al. 2019a which was the protologue for this species.

Macro-morphology - Basidiomata small. Pileus 8-25 $\mathrm{mm}$ diameter, convex to applanate, conchate, greyish when young, turns greyish-brown with maturity, surface entirely mattedtomentose, slightly hygrophanous, margin slightly transparent-striate. Lamellae moderately close, two tiers of lamellulae present, adnate, ventricose, $4 \mathrm{~mm}$ wide, pale greyish colour at first, become pink with age, all edges concolorous. Stipe 1-2.5 × 0.5-1 mm, reduced, lateral, greyish brown, slightly covered with fibrils, basal area covered with white mycelium. Thin Context, greyish. Odour absent. No distinctive taste.

Micro-morphology - Basidiospores 8-10.5 × 6-7.5 $\mu \mathrm{m}, \mathrm{Q}=1.25-1.61,5-6$ angled, heterodiametric. Basidia 4-spored, 20-34 × 8-11 $\mu \mathrm{m}$, clavate, clampless. Fertile Lamellar edge. Cheilocystidia, pleurocystidia and caulocystidia absent. Pileipellis a cutis formed with cylindric hyphae, terminal cells 40-65 ×5-7 $\mu \mathrm{m}$, slightly uplifted, cylindrical, non-gelatinised thin wall, minutely encrusted with pale brown pigments. In pileipellis Oleiferous hyphae and clamp connections present.

Ecology and Distribution - Saprotrophic, grow on decaying stumps of Castanopsis sp., on soil, rock, moss in forest dominated by Quercus sp. Recorded from Yunnan, China (He et al.2019a).

Entoloma sinuatum (Bull. ex Pers: Fr.) Kummer. Führ. Pilzk. (Zerbst): 97 (1871)

Following description is based on literature gathered from protologue (Fries \& Elias 1821) 
and Horak (1987).

Macro-morphology - Basidiomata medium-sized. Pileus 30-250 $\mathrm{mm}$ in diameter, conicoconvex first but expands to either convex or applanate, with or without broad low umbo, when become mature margin become irregularly concave, pale grey-livid or cream color, glabrous and smooth, fluffy or rugulose at centre. Lamellae moderately crowded, $1-3$ tiers of lamellulae, adnate or emarginate, narrowly segmentiform, appears in distinct yellow when young, turns into salmonyellow or to pink, the yellow colour retains for a long time towards margin of pileus, serrulate and concolorous edge. Stipe $40-150 \times 5-35 \mathrm{~mm}$, cylindrical, sometimes compressed, distinctly swollen base, white or grey, subconcolorous with pileus, pruinose apex, smooth downwards, fibrillose. Context firm, white. Smell strong, Taste unpleasant.

Micro-morphology - Basidiospores 8-11 × 7-9.5 $\mu \mathrm{m}, \mathrm{Q}=1.0-1.3,6$-angled. Basidia 4spored, clamped. Fertile Lamella edge. Cystidia absent. Pileipellis an ixo-cutis, narrow, cylindrical hyphae present, yellow-brown, 2-5 $\mu \mathrm{m}$ wide, intracellular pigment present, Clamps abundant.

Ecology and Distribution - Ectomycorrhizal. Grow solitary or in groups forming fairy rings. Fairly common in North America, Europe and the British Isles including Ireland. Except that $E$. sinuatum is recorded from Black Sea region, Turkey, Iran, and Yunnan in China (Li et al.2009, Xiao et al. 2012)

Chemical properties and special features - Pileipellis hyphae contain blue intracellular pigment in suprapellis and with brown cytoplasmic pigment in hypodermal hyphae. Stipitipellis hyphae present with blue intracellular pigment (Li et al. 2009, Xiao et al. 2012).

Entoloma yunnanense J.Z.Ying, Mycotaxon 54: 309 (1995)

Following description is based on the protologue in Ying (1995)

Macro-morphology - Basidiomata small. Pileus 9-15 mm in diameter. Hemispherical to subcampanulate, brown colour, fibrils present, squamulose. Lamellae emarginated, sub-decurrent. Stipe $55-80 \times 2 \mathrm{~mm}$, cylindrical, concolourous with pileus.

Micro - morphology - Basidiospores 12.6-16.2 × 7.2-10 $\mu \mathrm{m}$, sub-hyaline, 5-8 angled. Basidia 4-spored, 34-54 × 12.6-16 $\mu \mathrm{m}$. Pleurocystidia absent. Cheilocystidia 19.8-23.4 × 14.4-18 $\mu \mathrm{m}$, clavate.

Ecology and Distribution - Saprotrophic, Gregarious, grow on grounds. Recorded from Yunnan, China.

Table 1 Information on taxa and GenBank accession numbers of ITS based sequences of Entoloma specimens used for phylogenetic analysis

\begin{tabular}{llll}
\hline Name of the species & Voucher & Locality & $\begin{array}{l}\text { Gen Bank accession number } \\
\text { (ITS, 5.8S) }\end{array}$ \\
\hline E. azureosquamulosum & HKAS53408 & China & JQ410334 \\
E. stylophorum & GDGM25736 & China & JQ281480 \\
E. subtenuicystidiatum & GDGM 29246 & China & JQ320114 \\
E. praegracile & GDGM 29256 & China & JQ320107 \\
E. caespitosum & GDGM24025 & China & JQ281490 \\
E. mastoideum & GDGM28820 & China & JQ281476 \\
E. coelestinum & HMLD1659 & China & KC257434 \\
E. holmvassdalenense (Type) & O-F75311 & Norway & KM610321 \\
E. caeruleopolitum & RBG Kew K M 102319 & England & EU784210 \\
E. chalybaeum var. lazulinum & RBG Kew K M 90810 & England & EU784215 \\
E. insidiosum & L376 & Russia & KC898443 \\
E. virescens & - & Japan & AB509863 \\
E. hochstetteri & TL2573 & New Zealand & KP191941 \\
E. mengsongense (holotype) & HKAS90774 & China & KU131556 \\
E. petchii & HKAS56716 & China & JQ281485 \\
E. omiense & GDGM27563 & China & JQ281487 \\
E. inocephalum & LE262922 & Viet Nam & KC898449 \\
E. griseocyaneum & LE254351 & Russia & KC898444 \\
\hline
\end{tabular}


Table 1 Continued.

\begin{tabular}{|c|c|c|c|}
\hline Name of the species & Voucher & Locality & $\begin{array}{l}\text { Gen Bank accession number } \\
\text { (ITS, 5.8S) }\end{array}$ \\
\hline E. turci & 3882 & USA & JF907993 \\
\hline E. incanum & HKAS54614 & China & JQ281488 \\
\hline E. asprellum & RBG Kew K M 91347 & England & EU784206 \\
\hline E. anatinum & RBG Kew K M 58618 & England & EU784202 \\
\hline E. mougeotii & LE254352 & Russia & КС898446 \\
\hline E. serrulatum & HKAS 52713 & Iran & КC898446 \\
\hline Entoloma sp. & HKAS 52713 & China & KT833862 \\
\hline E. aff. kujuense & - & Japan & JQ410336 \\
\hline E. nitidum & - & Canada & AB509866 \\
\hline E. bloxamii & - & Canada & AY228340 \\
\hline E. flavidum & GDGM24473 & China & EF530938 \\
\hline E. chalybaeum & LE254353 & Russia & JQ281481 \\
\hline E. abortivum & GDGM27313 & China & КС898445 \\
\hline E. shandongense & CUH AM109 & India & KP241852 \\
\hline E. conferendum & HKAS48953 & China & KP241852 \\
\hline E. lampropus & LE9121 & Russia & JQ281484 \\
\hline E. subaraneosum & GDGM 28823 & China & KC898378 \\
\hline E. pallidocarpum & GDGM 28828 & China & JQ320113 \\
\hline E. sinuatum & AFTOL-ID 524 & USA & JQ3201 \\
\hline Lyophyllum decastes & Lc42 T5P & Switzerland & AF357060 \\
\hline L. decastes & 901016 & Sweden & HM572546 \\
\hline E. ravinense & PSC3331 & Australia & KX387622 \\
\hline E. atricolor & LE 295001 & Vietnam & KY777496 \\
\hline E. atricolor & LE295002 & Vietnam & KY777497 \\
\hline E. bulakhae & LE 253787 & Russia & NR_158425 \\
\hline E. bidupense & LE 262935 & Vietnam & MF476906 \\
\hline
\end{tabular}

Phylogram formed from ML analysis based on combined ITS sequence data of Entoloma species available in Yunnan, China. Related sequences were acquired from GenBank. Forty-four taxa are included in the sequence analyses, which comprise 1032 number of characters with gaps. Lyophyllum decastes (901016) and Lyophyllum decastes (Lc42T5P) were used as the out group taxa. The best scoring RAxML tree with a final likelihood value of -13967.009706 is presented. The matrix had 795 distinct alignment patterns, with $37.61 \%$ of undetermined characters or gaps. Estimated base frequencies were as follows; $\mathrm{A}=0.281044, \mathrm{C}=0.188343, \mathrm{G}=0.193093, \mathrm{~T}=$ 0.337520 ; substitution rates $\mathrm{AC}=1.527188, \mathrm{AG}=3.374662, \mathrm{AT}=2.188792, \mathrm{CG}=0.731264, \mathrm{CT}$ $=4.565623, \mathrm{GT}=1.000000$; gamma distribution shape parameter $\alpha=0.817722$.

The maximum parsimonious dataset consisted of 353 constant, 542 parsimony-informative and 137 parsimony-uninformative characters. The parsimony analysis of the data matrix resulted in the maximum of two equally most parsimonious trees with a length of 3210 steps $(C I=0.393$, RI= $0.482, \mathrm{RC}=0.189, \mathrm{HI}=0.607)$ in the tree. $\mathrm{RAxML} \&$ maximum parsimony bootstrap support values $\geq 50 \%(\mathrm{BT})$ are shown respectively near the nodes. The type species are in bold.

\section{Discussion}

The morphological characteristics of reported Entoloma species share similar features with few exceptions. Cap size is one of the major morphological features with appreciable variation among species studied. It ranges from $3.5 \mathrm{~mm}$ in E. mengsongense to $250 \mathrm{~mm}$ in E. sinuatum.

A myriad of cap colors were observed among the species we studied vary from pale to shades of deep blue and brown color. Cap shape is another feature noticed with appreciable variation among different species and range from conical, convex, plano-convex, campanulate, hemispherical to broadly convex. Margin of the cap may in rolled when young, then involute and later deflexed. Lamella attachment in Entoloma species vary from adnexed, narrowly adnate to 
emarginate and color may vary from white, greyish white to pink. Stipe of Entoloma sporocarps can be solid, stuffed or fibrillose and size also varies greatly among various species of Entoloma.

Of the various micro-morphological features, the spore size and spore shape of Entoloma species vary greatly and those characteristics have particular importance in species segregation. In the present study the smallest spores were observed in E. mengsongense $(4-8 \times 4-6 \mu \mathrm{m})$ and the largest were in E. yunnanense. The color of the spores is usually pink and varies from reddish pink to brownish pink. Spore shape is also important and found varying from cuboid, heterodiametric, pentagonal, heptagonal or even with irregular edges. Spores of most species are hyaline and thinwalled.

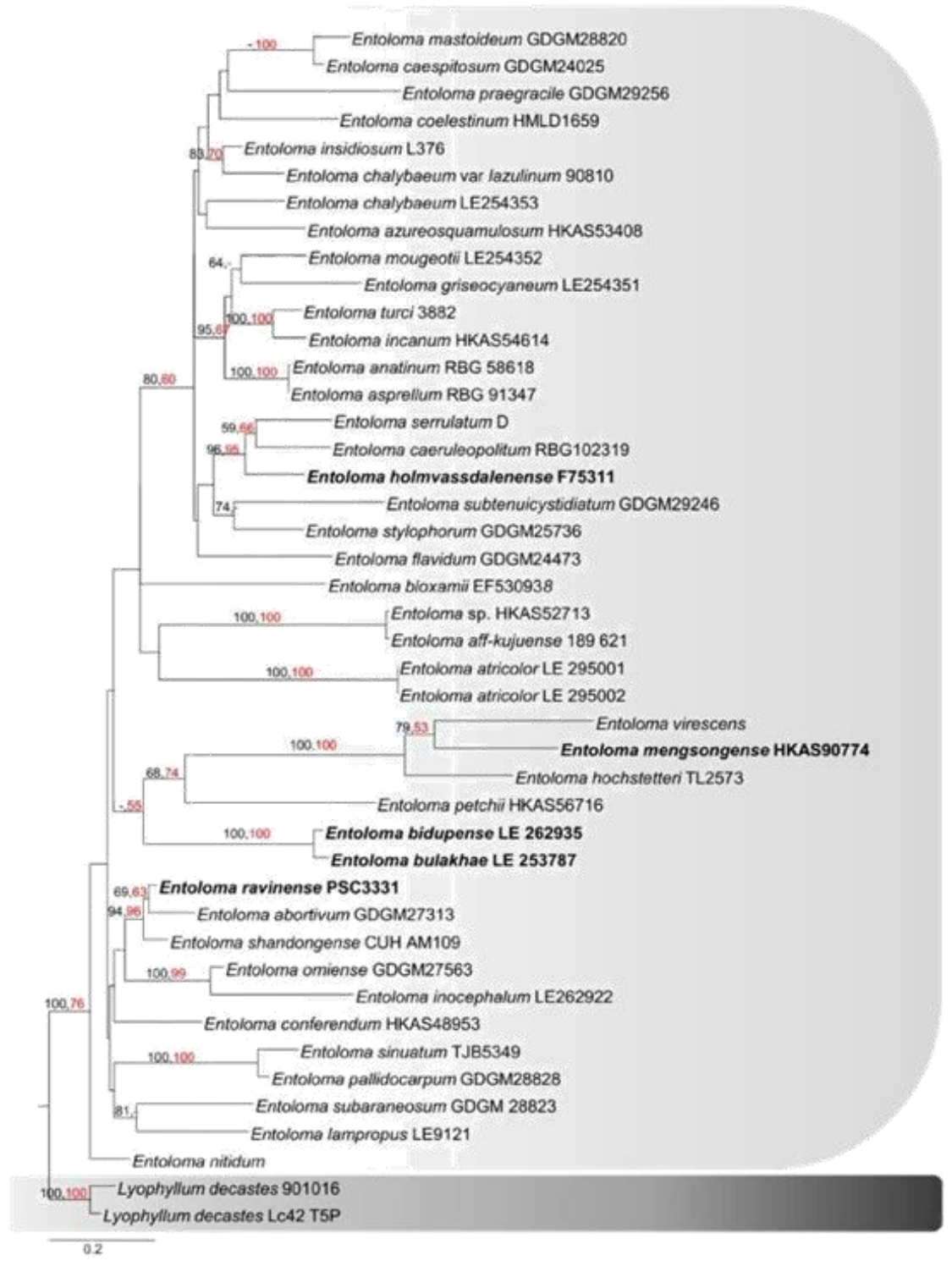

Fig. 1 - Phylogenetic tree obtained from RAxML analyses that shows the phylogenetic positioning of different Entoloma species based on the sequences of ITS data. Bootstrap support values for maximum parsimony (MP, left) and maximum likelihood (ML, right) greater than $50 \%$ and are given at the nodes. The tree is rooted with Lyophyllum decastes and the type species are indicated in black bold.

The size and shape of basidia in Entoloma species studied were almost uniform and clavate. But this characteristic does not value much at the species level recognition. The number of sterigmata is found to vary with species, though majority were 4-sterigmate while 1, 2, 3-were also 
observed. Presence or absence of cheilocystidia is important and observed only in E. yunnanense. Oleiferous hyphae, pileipellis and stipitipellis is discussed with relevance to E. mengsongense and Oleiferous hyphae was found in E. caeruleoflavum, E. gregarium, E. pleurotoides and E. reductum. Cheilocyctedia and Pleurocystidia had been only recorded from E. yunnanense among species recorded from Yunnan. Specific odor and taste has been recorded from E. mengsongense whilst rest of the Entoloma species discussed in the study has no distinctive odor and taste.

Distribution and ecology of Entoloma species found in Yunnan province, China, are mostly saprotrophic and found to occur in decomposing matter of leaf litter and other debris. E.sinuatum and E.caeruleoflavum are found only species found to form ectomycorrhizal association. Though many Entoloma species were reported from China, many of them are misidentified and lack of voucher specimens (Xiao et al. 2012).

Economically this genus is of little value and E. abortivum is the only species coming under the edible category while none were found to have medicinal value among species recorded from Yunnan. But, many species of Entoloma were reported as edible and medicinal worldwide. Entoloma aprile, E. argyropus, E. crassipes, E. madidum and E. microcarpum are valued as edible mushrooms in many regions of world (Hall 2016) while E. nitidum is well known for its antitumor properties that inhibits the growth of Sarcoma 180 and Ehrlich solid carcinoma in mice by 60 to 70\%, respectively (Ohtsuka et al. 1973, Isiloglu et al. 2010, Ainsworth et al. 2018). Entoloma srtictius and E. murrayi are highly toxic. Knowledge on biochemical properties of the species are important to utilize them properly for food, medicine and in industry.

\section{Conclusion}

A comprehensive review of species of Entoloma in Yunnan, China is provided. It details the preference and variations of individual species on habit, habitat, distribution, macro and micromorphological and molecular features. Data on ecology and morphology of genus Entoloma though well known, little is known about its chemical attribute. Extended chemical studies are necessary to know the chemical attributes of the species which in turn will pave way for better utilization as food and medicine.

\section{Acknowledgement}

Kevin D. Hyde thanks the Chinese Academy of Sciences, project number 2013T2S0030, for the award of Visiting Professorship for Senior International Scientists at Kunming Institute of Botany. This study was funded by the Chinese Ministry of Science and Technology, under the 12th five-year National Key Technology Support Program (NKTSP) 2013BAB07B06 for integration and comprehensive demonstration of key technologies on Green Phosphate-Mountain Construction; the CGIAR Research Program 6: Forest, Trees and Agroforestry; Thailand Research Fund grant Taxonomy, Phylogeny and Biochemistry of Thai Basidiomycetes (BRG 5580009); the National Research Council of Thailand NRCT) projects - Taxonomy, Phylogeny and Cultivation of Lentinus species in northern Thailand (NRCT/55201020007); and Mae Fah Luang University, project Taxonomy, Phylogeny and Cultivation of Lentinus species in Northern Thailand (MFU/54 10102 $0048)$.

We would like to acknowledge Dr. C.K. Pradeep, Senior Scientist and Head, Microbiology Division, Jawaharlal Nehru Tropical Botanic Garden and Research Institute for his suggestions and comments during the preparation of the article.

\section{Reference}

Ainsworth AM, Douglas B, Suz LM. 2018 - Big Blue Pinkgills formerly known as Entoloma bloxamii in Britain: E. bloxamii s. str., E. madidum, E. ochreoprunuloides forma hyacinthinum and E. atromadidum sp. nov. Field Mycology 19, 5-14.

Co-David D, Langeveld D, Noordeloos ME. 2009 - Molecular phylogeny and spore evolution of Entolomataceae. Persoonia 23,147-176. 
Ediriweera AN, Karunarathna SC, Xu J, Mortimer PE. 2017 - Entoloma mengsongense sp. nov. (Entolomataceae, Agaricales), a remarkable blue mushroom from Yunnan Province, China. Turkish Journal of Botany 41, 505-515.

Fries, Elias M. 1821 - Systema Mycologicum, Lund, Officina Berlingiana 1,197

Gates GM, Noordeloos M. 2007 - Preliminary studies in the genus Entoloma in Tasmania-I. Persoonia 19, 157-226.

Gates GM, Noordeloos M. 2009 - Preliminary studies in the genus Entoloma in Tasmania-II. Cryptogamie Mycologie 30, 07-140.

Gates GM, Hortan BM, Noordeloos M. 2009 - A new Entoloma (Basidiomycetes, Agaricales) from Tasmania. Mycotaxon 107, 175-179.

Gargano ML, Mandracchia G, Saitta A, Venturella G. 2011 - Two rare Northern Entoloma species observed in Sicily under exceptionally cold weather conditions. The Scientific World 2012: 95722.

Hall LR. 2016 - A list of putative edible and medicinal ectomycorrhizal mushrooms. Technical Report.

He X, Jiang Z, Li T. 2010 - Three species of white Entoloma new to China. Mycosystema 29, 920923.

He XL, Horak E, Wang D, Li TH et al. 2019a - Descriptions of five new species in Entoloma subgenus Claudopus from China, with molecular phylogeny of Entoloma s.1. MycoKeys 61, $1-26$.

He M, Zhao R, Kirk PM. 2019b - Notes, outline and divergence times of Basidiomycota. Fungal Diversity 99, 105-367.

Hesler LR. 1967 - Entoloma (Rhodophyllus) in Southeastern North America. Beihefte zur Nova Hedwigia 23, 1-220.

Horak E. 1987 - “Agaricales from Yunnan China I”. Nippon Kingakukai Kaiho. 28, 171-88.

Isiloglu M, Sermenli HB, Senol A, Isler M. 2010 - Entoloma mushroom poisonings in Mediterranean Turkey. Turkish Journal of Botany 35, 247-249.

Karstedt F, Capelari M. 2013 - Inocephalus (Entolomataceae, Agaricales) from Sao Paulo State, Brazil. Nova Hedwigia 96, 279-308.

Kasuya T, Takehashi S, Hoshino T, Noordeloos ME. 2010 - Entoloma aprile (Agaricales, Entolomataceae) new to Japan, with notes on its mycorrhiza associated with Populus maximowiczii in cool-temperate deciduous forests of Hokkaido. Sydowia 62, 205-223.

Largent DL. 1994 - Entolomatoid Fungi of the Pacific Northwest and Alaska. Eureka, CA, USA: Mad River Press.

Li CH, Li TH. 2009 - A new Entoloma species (Entolomataceae, Agaricales) from Hainan Island. Mycosystema 28, 641-643.

Li TH, Li CH, Shen YH. 2009 - Two new blue species of Entoloma (Basidiomycetes, Agaricales) from South China. Mycotaxon. 107, 405-412.

Manimohan P, Joseph AV, Leelavathy KM. 1995 - The genus Entoloma in Kerala State, India. Mycological Research 99, 1083-1097.

Manimohan P, Noordeloos ME, Dhanya AM. 2006 - Studies on the genus Entoloma (Basidiomycetes, Agaricales) in Kerala state, India. Persoonia 19, 45-93.

Noordeloos ME, Morozova OV. 2010 - New and noteworthy Entoloma species from the Primorsky Territory, Russian Far East. Mycotaxon 112, 231-255.

Noordeloos ME. 2004 - Entoloma s.1. Fungi Europaei, vol. 5a.Edizione Candusso, Italy.

Noordeloos ME, Hausknecht A. 2007 - The genus Entoloma (Basidiomycetes, Agaricales) of the Mascarenes and Seychelles. Fungal Diversity 27, 111-144.

Ohtsuka S, Ueno S, Yoshikumi C, Hirose F et al. 1973 - Polysaccharides having an anticarcinogenic effect and a method of producing them from species of Basidiomycetes. UK Patent 1331513.

World Data Lab. 2020 - Available from World data info https://www.worlddata.info/asia/china/climate-yunnan.php (Accessed September 2020) 
Xiao LH, Li TH, Zi DJ, Shen YH. 2012 - Four new species of Entoloma s.1. (Agaricales) from southern China. Mycological progess 11, 915-952.

Xiao LH, Horak E, Li TH, Peng WH, Gan BC. 2015 - Two new cuboid-spored species of Entoloma s.l. (Agaricales, Entolomataceae) from southern China. Cryptogamie, Mycologie $36,237-249$

Ying JZ. 1995 - New and noteworthy Agarics from China II. New species of Entoloma. Mycotaxon 54, 309-313.

Zhang W, Li TH. 2001a - A new species of Entoloma from Nanling National nature reserve. Mycosystema 21, 483-484.

Zhang W, Li TH. 2001b - A new subgenus and a new species of Entoloma. Mycosystema 21, 153155. 